\title{
Evaluación de la Eficiencia de la Transferencia de Embriones Interespecie entre Alpacas y Lamas Obtenidos por Ovulación Simple
}

\author{
Evaluation of the EFFiciency of InTERSPECIES Embryo Transfer betweEn AlpaCaS \\ and Llamas Obtained through Single Ovulation
}

\author{
Joel Pacheco ${ }^{1,2}$, Víctor Vélez ${ }^{1}$, Danilo Pezo ${ }^{1}$
}

\section{Resumen}

\begin{abstract}
El presente estudio tuvo como objetivo evaluar la eficiencia de recuperación de embriones de ovulación simple, la calidad de los embriones y la fertilidad de la transferencia embrionaria interespecie. Se utilizaron 9 alpacas donadoras y 40 llamas receptoras. Se les hizo el seguimiento ecográfico para determinar la presencia de folículos ovulatorios. Las donadoras fueron cubiertas mediante monta natural y el lavado de los cuernos uterinos se realizó a los 7 días de la cópula. Luego del lavado uterino se aplicó cloprostenol a las donadoras para inducir luteólisis y reiniciar una nueva onda folicular. Se realizaron cuatro lavados con un intervalo de 15 días entre lavados. Las transferencias de los embriones se hicieron en el día 7 (grupo I) y 6 (grupo II) pos-inducción de la ovulación con buserelina a las receptoras. Se recolectó el 58.3\% de los embriones. El 57.2, 23.8 y $19.0 \%$ de los embriones fueron de grado 1,2 y 3 , respectivamente. No hubo embriones de grado 4. La fertilidad a los 21 días de la transferencia fue de 33.3 y $11.1 \%$ para los grupos 1 y 2 , respectivamente (promedio: $20.0 \%$ ), sin registros de mortalidad embrionaria en los controles a los 90 y 150 días de la transferencia.
\end{abstract}

Palabras clave: embriones, transferencia interespecie, ovulación simple

\footnotetext{
${ }^{1}$ Centro de Investigación IVITA-Maranganí, Facultad de Medicina Veterinaria, Universidad Nacional Mayor de San Marcos, Cusco, Perú

${ }^{2}$ E-mail:mvz_joelpc@hotmail.com
}

Recibido: 6 de junio de 2015

Aceptado para publicación: 7 de octubre de 2015 
This study aimed to evaluate the efficiency of embryo recovery in single ovulation, the quality of the embryos and the interspecies fertility through embryo transfer. Nine donor alpacas and 40 recipient llamas were used. Ultrasonography of the ovaries was done to determine the presence of an ovulatory follicle. Donors were naturally mated and washing of uterine horns was performed at 7 days post-breeding. After collecting the embryos, cloprostenol was injected to donors to induce luteolisis and reinitiate a new follicular wave. Four washes were performed with intervals of 15 days. The transfer of fresh embryos was done on day 7 (group 1) or 6 (group 2) after the induction of ovulation with buserelin to the recipients. The results showed that $58.3 \%$ of embryos were collected. Among them, 57.2, 23.8 and 19.0\% were of grade 1, 2 and 3 respectively. None embryos were of grade 4 . Fertility on day 21 after the transfer of embryos was 33.3 and $11.1 \%$ for groups 1 and 2 respectively (mean: $20 \%$ ), without recording embryo mortality in controls at 90 and 150 days post-transfer.

Key words: embryos, interspecies transfer, simple ovulation

\section{INTRODUCCIÓN}

La transferencia embrionaria es una biotecnología alternativa para realizar mejoramiento genético utilizando individuos superiores. Esta técnica viene siendo estudiada y mejorada con el objeto de utilizarla en los camélidos sudamericanos, dada su particular fisiología reproductiva (Huanca, 2005). Al igual que en otras especies, la técnica de multiovulación y transferencia de embriones fue desarrollada en alpacas (Bourke et al., 1995); sin embargo, existe una respuesta muy variable a los protocolos de superovulación en los camélidos, siendo así que solo el $33.5 \%$ de las hembras responden a los estímulos hormonales para el desarrollo de múltiples folículos, además de obtenerse ovocitos con desarrollo anormal y deficiente maduración nuclear y citoplasmática, lo que afecta las tasas de fertilización (Vaughan et al., 2013).

La transferencia de embriones se realiza mediante la técnica no quirúrgica el día 6 o 7 poscópula, depositando el embrión directamente en el cuerno ipsilateral al folículo ovulatorio (Carnero et al., 2011, Trasorras, 2012; Sumar, 2013), donde pareciera que la transferencia del embrión en el día 6 es más efectivo, pues permite un mayor tiempo para el reconocimiento del embrión, especialmente considerando que la secreción de PGF $_{2 \alpha}$ se inicia el día 7 (Trasorras et al., 2010).

Actualmente se viene evaluando la técnica de lavado uterino en alpacas con ovulación simple, que no requiere un tratamiento previo de superovulación y, por lo tanto, es de menor costo, lográndose hasta $70 \%$ de efectividad de colección mediante esta técnica (Huanca et al., 2012; Vaughan, 2012). Con este método, se pueden realizar lavados sucesivos en las hembras donantes con periodos de descanso, obteniéndose $50 \%$ de embriones de calidad «excelente» (Grado 1) y $25 \%$ de calidad «bueno» (Grado 2), lo que significa que el $75 \%$ de embriones recuperados serían transferibles (Pineda et al., 2012).

La transferencia de embriones interespecie es factible de realizar entre ciertas especies, tal como fue demostrado entre dromedarios y camellos bactrianos (NiasariNaslaji et al., 2009), y es una opción para lograr mejores tasas de gestación al transferir embriones de alpaca en llamas receptoras. De esta manera, se utiliza la mayor habilidad materna de la llama y lograr tasas de preñez de hasta 70\% (Sumar et al., 2012). 
Las crías resultantes nacen con pesos de 3.1 a $3.3 \mathrm{~kg}$, pesos superiores a aquellos de crías alpaca nacidas de alpacas receptoras. Además, llegan a los $33.4 \mathrm{~kg}$ a los 6 meses a diferencia de los $22.2 \mathrm{~kg}$ que alcanzan las crías nacidas de alpacas receptoras (Huanca et al., 2012; Sumar et al., 2012).

Los objetivos de este estudio fueron evaluar la eficiencia del lavado uterino para recuperar embriones de ovulación simple en alpacas al día 7 de la cópula, evaluar la calidad de estos embriones y determinar la fertilidad de la transferencia de embriones los días 6 y 7 en llamas receptoras, así como la natalidad y pesos al nacimiento.

\section{Materiales y Métodos}

\section{Lugar de Ejecución y Animales}

El trabajo fue desarrollado durante los meses de marzo y abril, época reproductiva de los camélidos sudamericanos, en el fundo La Raya de la Estación Experimental del Centro de Investigación IVITA, Maranganí, Cusco, Perú, a una altitud de $4200 \mathrm{msnm}$. Se usaron nueve alpacas Huacaya adultas de reconocida eficiencia reproductiva como donadores de embriones. Asimismo, se usaron 40 llamas adultas con historial reproductivo como receptoras. Los animales fueron alimentados en pasturas naturales.

Se realizó un examen ecográfico a las donadoras y receptoras después del periodo de descanso posparto para determinar la presencia de folículos preovulatorios (>7 mm). Las receptoras se dividieron en dos grupos de 20 animales para la transferencia de los embriones en los días 7 (Grupo 1) y 6 (Grupo 2) poscópula, respectivamente.

\section{Tratamientos}

En el caso de las donadoras se realizó el empadre natural (día 0) y a los 7 días se realizó el lavado uterino mediante la técnica no quirúrgica, usando medio de lavado EmFlush II (Immunosystems). Luego se aplicó un análogo de prostaglandina $\mathrm{F}_{2 \alpha}$ para terminar la actividad funcional del cuerpo lúteo $\mathrm{y}$ asegurar el desarrollo de otra onda folicular. En el día 15 se realizó un segundo empadre y 7 días después se realizó el segundo lavado uterino. Este procedimiento se repitió hasta realizar cuatro lavados uterinos por donadora.

En las receptoras se aplicó GnRH como inductor de ovulación el mismo día del lavado uterino de las donadoras para el Grupo 1 y un día después para el Grupo 2. La transferencia de los embriones frescos se realizó de acuerdo a los grupos de receptoras (6 o 7 días de la inducción de la ovulación). Los embriones fueron depositados en el cuerno izquierdo en todas las transferencias. La concepción se evaluó el día 21, 90 y 150 de la transferencia embrionaria usando un ecógrafo Aloka SSD250 y un transductor de $5 \mathrm{MHz}$ mediante ecografía transrectal.

Como inductor de ovulación en las llamas se utilizó $0.0082 \mathrm{mg}$ de acetato de buserelina vía intramuscular y para lograr la luteólisis de los cuerpos lúteos en las donadoras se utilizó cloprostenol, $0.048 \mathrm{mg}$. En el lavado uterino se empleó una sonda de Foley, $100 \mathrm{~mL}$ de medio de lavado por cuerno en dos lavados sucesivos y se recuperó el fluido en un filtro EmCon (Agtech ${ }$, Holanda).

Todos los lavados y transferencias fueron hechos por la misma persona. Asimismo, los lavados de cada etapa fueron realizados en un solo día.

\section{Evaluación de Embriones}

Los embriones fueron evaluados con apoyo de un estereoscopio con platina temperada a $25{ }^{\circ} \mathrm{C}$. Los embriones fueron colocados en una gota de medio de mantenimiento temperado (Singro Holding, Bioniche®) y la evaluación se realizó usando la escala mencionada en el manual de la International Embryo Transfer Society - IETS (Stringfellow y Givens, 2011), transfiriendo solo embriones de los grados 1 y 2 . 
Cuadro 1. Calidad de embriones de alpaca de acuerdo al número de lavado uterino

\begin{tabular}{cccccc}
\hline \multirow{2}{*}{$\begin{array}{l}\text { Número } \\
\text { de } \\
\text { lavado }\end{array}$} & \multicolumn{5}{c}{ Calidad de embrión (\%) } \\
\cline { 2 - 6 } & 1 & 2 & 3 & 4 & Prom. \\
\hline $1^{\circ}$ & $2 / 7$ & $3 / 7$ & $2 / 7$ & 0 & $7 / 9$ \\
$2^{\circ}$ & $5 / 6$ & $1 / 7$ & 0 & 0 & $6 / 9$ \\
$3^{\circ}$ & $3 / 5$ & $1 / 5$ & $1 / 5$ & 0 & $5 / 9$ \\
$4^{\circ}$ & $2 / 3$ & 0 & $1 / 3$ & 0 & $3 / 9$ \\
\hline Total & $12 / 21$ & $5 / 19$ & $4 / 15$ & 0 & $21 / 36$ \\
\hline
\end{tabular}

\section{Análisis Estadístico}

Se realizó la evaluación estadística mediante la prueba del Chi Cuadrado y medidas de tendencia central, usando el programa SAS (SAS, 2002).

\section{Resultados}

La tasa de recuperación de embriones fue disminuyendo con el número de lavados realizados. Así, se logró el 77.7, 66.4, 55.5 y $33.3 \%$ de recuperación para el $1^{\circ}, 2^{\circ}, 3^{\circ}$ y $4^{\circ}$ lavado, respectivamente. Algunas donadoras no dieron ningún embrión en tanto que otras produjeron hasta tres embriones. El 57.2\% de los embriones fue de grado 1 , el $23.8 \%$ de grado 2 , el $19.0 \%$ de grado 3 , no habiendo embriones de grado 4 (Cuadro 1).

La fertilidad total (ambos grupos) fue de $20.0 \%$ (Cuadro 2). La natalidad fue del $20 \%$, obteniéndose 3 crías de 15 embriones transferidos (2 embriones no fueron transferidos pues sufrieron daño durante la transferencia en campo). Los pesos al nacimiento de las crías fueron de $7.5,6.5$ y $7.5 \mathrm{~kg}$, respectivamente (promedio de $7.2 \mathrm{~kg}$ ).

\section{Discusión}

Los porcentajes de recuperación al primer y segundo lavado concuerdan con lo descrito por otros autores (Huanca et al., 2012; Vaughan, 2012), donde se obtiene hasta $70 \%$ de recuperación de embriones a los 7 días de la cópula. Sin embargo, las tasas de recuperación no se mantienen en los lavados sucesivos en el presente estudio, indicando una importante merma en la respuesta a la repetición de los lavados uterinos.

La calidad de los embriones fue mayoritariamente de grado 1 , seguido de los embriones de grado 2, llegando a obtenerse $81 \%$ de embriones transferibles, resultados similares a lo descrito por Pineda et al., (2012), quienes obtuvieron $75 \%$ de embriones transferibles. Es importante resaltar que la frecuencia de embriones transferibles se mantuvo a través de los cuatro lavados.

Solo se llegó a recuperar 21 embriones en los 36 lavados uterinos, lo cual representa el 58.3\% de tasa de recuperación, porcentaje inferior a los resultados de $72.3 \%$ de recuperación reportados por Huanca et al. (2014a). Estas diferencias podrían atribuirse a la alimentación de pasturas naturales y sin suplementación adicional de las donadoras, dado que se trataba de reproducir las condiciones de manejo del productor promedio. El factor nutricional es un aspecto clave en la respuesta ovulatoria de la donadora, donde se ha observado mejores respuestas ovulatorias en animales mantenidos en pasturas cultivadas respecto a las hembras mantenidas en pasturas naturales (Gonzales et al., 2014).

Se transfirieron 15 (71.4\%) embriones del total de recuperados, los cuales corresponden a los embriones de los grados 1 y 2 considerados de calidad transferibles (Stringfellow y Givens, 2011). Los valores obtenidos fueron similares a los reportados por Huanca et al. (2014a), quienes trabajaron con lavados uterinos en alpacas de ovulación simple. 
Cuadro 2. Resultados de la transferencia de embriones de alpaca en llamas receptoras a los 6 y 7 días post-inducción de ovulación

\begin{tabular}{lcccccc}
\hline & \multicolumn{2}{c}{7 días } & \multicolumn{2}{c}{6 días } & \multicolumn{2}{c}{ Total } \\
\cline { 2 - 7 } & $\mathrm{n}$ & $\%$ & $\mathrm{n}$ & $\%$ & $\mathrm{n}$ & $\%$ \\
\hline Lavados uterinos (n) & 18 & & 18 & & 36 & \\
Recuperación de embriones & 10 & 55.5 & 11 & 61.1 & 21 & 58.3 \\
Embriones transferidos & 6 & 60.0 & 9 & 81.0 & 15 & 71.4 \\
Fertilidad & 2 & $33.3^{\mathrm{a}}$ & 1 & $11.1^{\mathrm{a}}$ & 3 & 20.0 \\
\hline
\end{tabular}

Los porcentajes de fertilidad fueron bajos (Cuadro 2) en ambos grupos e inferiores a otros reportes que señalan $37.5 \%$ de éxito en transferencias a receptoras a los 7 días de inducción de la ovulación (Huanca et al., 2014b). La diferencia en fertilidad entre grupos, si bien no es estadísticamente significativa debido al pequeño número de animales, se pudo observar una mejor tasa de concepción a los 7 días de la ovulación, resultado que difiere de los resultados de Trasorras et al. (2010), quienes obtienen una mayor fertilidad con transferencias a los 6 días de la ovulación. Otro factor que pudo haber influido en los resultados fue que, en todos los casos, el embrión fue depositado en el cuerno uterino izquierdo, tal como lo describen Carnero et al. (2011), quienes indican que el mejor resultado se obtiene depositando el embrión en dicho cuerno, indistintamente del lado donde ocurrió la ovulación.

Posiblemente, debido al plano nutricional en que se encontraban las donadoras y las receptoras, las tasas de natalidad y los pesos al nacimientos de las crías fueron igualmente inferiores a los obtenidos por Huanca et al. (2014b) y Vaughan (2012) en el primer caso, y Sumar et al. (2012) en el segundo.

\section{Conclusiones}

- La tasa de recuperación de embriones de alpaca en ovulación simple disminu- ye a través del número de lavados uterinos; sin embargo, persiste la buena calidad de los embriones recuperados.

- No se encontraron diferencias estadísticas por efecto del día de la transferencia de embriones (días 6 o 7) sobre la fertilidad, natalidad y peso al nacimiento de las crías, posiblemente debido al reducido número de embriones disponibles.

\section{Literatura Citada}

1. Bourke DA, Kyle CE, McEvoy TG, Young P, Adam CL. 1995. Recipient synchronization and embryo transfer in South American camelids. Theriogenology 43: 171 (Abstract).

2. Carnero S, Huanca W, Cordero A, Vásquez M, Huanca T. 2011. Transferencia embrionaria ipsilateral y contralateral a la posición del cuerpo lúteo y supervivencia embrionaria en llamas. Rev Inv Vet Peru 22: 114-120. doi: 10.15381/rivep.v22i2.278

3. Gonzáles ML, Huanca T, Cárdenas O, Mamani RH, Sapana R, Huanca W. 2014. Efecto de la edad y el estado nutricional en la respuesta ovárica y calidad de embriones de alpacas. En: Mem XXXVII Reunión Científica Anual de la Asociación Peruana de Producción Animal. Abancay: APPA. p 235-237. 
4. Huanca W. 2005. Aplicación de biotecnologías reproductivas en especies domésticas y silvestres de camélidos sudamericanos. Agrociencia 9(1-2): 505-509.

5. Huanca T, Sumar J, Naveros M, Cárdenas O, Gonzáles M, Mamani R. 2012. Twin reciprocal embryo transfer between alpacas and llamas. In: ICAR 2012. Satellite Meeting on Camelid Reproduction. Vancouver, Canada.

6. Huanca T, Mamani RH, Cárdenas $O$, Gonzáles ML, Sapana R. 2012. Evaluación del peso al nacimiento, destete, al año de edad y curva de crecimiento de alpacas y llamas cría nacidas por transferencia de embriones interespecies. Spermova 2(1): 44-46.

7. Huanca T, Gonzáles M, Mamani-Cato $\mathrm{RH}$, Cárdenas O, Sapana $R, y$ Naveros M. 2014a. Evaluación de la recuperación de embriones en alpacas y llamas donadoras simples y superestimuladas. En: Mem XXXVII Reunión Científica Anual de la Asociación Peruana de Producción Animal. Abancay: APPA. p 252-254.

8. Huanca T, Gonzáles M, Mamani-Cato RH, Cárdenas O, Sapana R, Naveros M. 2014b. INIA: Avances en la transferencia de embriones de camélidos domésticos. En: Mem XXXVII Reunión Científica Anual de la Asociación Peruana de Producción Animal. Abancay: APPA. p 31-34.

9. Niasari-Niaslaji A, Nikjou D, Skidmore JA, Moghiseh A, Mostafaey M, Razavi K, Moosavi-Movahedi A. 2009. Interspecies embryo transfer in camelids: the birth of the first bactrian camel calves (Camelus bactrianus) from dromedary camels (Camelus dromedarius). Reprod Fert Dev 21: 333-337. doi: 10.1071/RD08140
10. Pineda J, Pozo A, Huanca T, Naveros ML. 2012. Recuperación sucesiva de embriones y retorno folicular post lavado en alpacas (Vicugna pacos) Huacaya donadoras naturales. Spermova 2(1): 53-54.

11. Stringfellow D, Givens D. 2011. Manual de la Sociedad Internacional de Transferencia de Embriones. $4^{\mathrm{a}}$ ed. Illinois, EEUU: International Embryo Transfer Society. $185 \mathrm{p}$.

12. Sumar J, Arellano P, Montenegro V, Londoñe P, Picha Y, Rodriguez C, Sanchez D, Torres R. 2012. Reciprocal embryo transfer in alpacas and llamas. In: ICAR 2012. Satellite Meeting on Camelid Reproduction. Vancouver, Canada.

13. Sumar J. 2013. Embryo transfer in domestic South American camelids. Anim Reprod Sci 136: 170-177. doi: 10.1016/ j.anireprosci.2012.10.029

14. Trasorras V, Chaves MG, Neild D, Gambarotta M, Aba M, Agüero A. 2010. Embryo transfer technique: factors affecting the viability of the corpus luteum in llamas. Anim Reprod Sci 121: 279-285. doi: 10.1016/j.anireprosci. 2010.06.004

15. Trasorras VL. 2012. Producción de embriones in vivo e in vitro en camélidos sudamericanos. Spermova 1: 19-21.

16. Vaughan JL. 2012. Embryo transfer in alpacas. In: ICAR 2012. Satellite Meeting on Camelid Reproduction. Vancouver, Canada.

17. Vaughan J, Mihm M, Wittek T. 2013. Factors influencing embryo transfer success in alpacas. A retrospective study. Anim Reprod Sci 136: 194-204. doi: 10.1016/j.anireprosci.2012.10.010 